\title{
A Hospital Based Comparative Clinical study between Mometasone Furoate and Fluticasone Furoate Nasal Spray for Allergic Rhinitis
}

\author{
${ }^{1}$ AsthomiJamoh, \\ ${ }^{I}$ senior Medical Officer, Bolung Phc, Lower Dibangvalley, Arunachal Pradesh \\ Asthomi Jamohsenior Medical Officer, Bolung Phc, Lower Dibang Valley, Arunachal Pradesh
}

\begin{abstract}
Introduction: Allergic rhinitis is an inflammatory disorder of the nose which occurs when the membranes lining the nose become sensitised to allergens. Although not classed as a life-threatening disease, allergic rhinitis can reduce the patient's quality of life. Mometasonefuroate is a glucocorticosteroid used topically to reduce inflammation of the skin or in the airways. It is a prodrug of the free form mometasone. Fluticasonefuroate is a synthetic corticosteroid with a very high affinity for the glucocorticoid receptor and has a potent antiinflammatory action.
\end{abstract}

Aim: Tocompare clinicaleffects between mometasonefuroate and fluticasonefuroate nasal spray for allergic rhinitis

Results: Two hundred fifty-nine patients were valid for efficacy. Mometasonefuroate was significantly more effective than placebo and was not different from fluticasonefuroate. The study was also physician-evaluated for total nasal symptoms. Overall, mometasonefuroate was at least as effective as fluticasonefuroate at equivalent doses.

Conclusion:Mometasonefuroate and fluticasonefuroate adequately controlled symptoms of perennial rhinitis and were well tolerated.

Source Of Support : Nil

Conflict Of Interest: Nil

\section{Introduction}

Allergic rhinitis is an inflammatory disorder of the nose which occurs when the membranes lining the nose become sensitised to allergens. Although not classed as a life-threatening disease, allergic rhinitis can reduce the patient's quality of life. Chronic rhinosinusitis with nasal polyps is a condition represented with inflammatory changes throughout the nose and sinuses from a group of disorders which all lead to swelling and overgrowth of the nasal mucosa ${ }^{1}$. Allergen avoidance is essential in the management of allergic rhinitis. Drug treatment may be necessary to prevent and control frequent or persistent symptoms, especially if nasal obstruction/polyps are present ${ }^{2}$. Intranasal corticosteroids are considered more effective than oral antihistamines at relieving most nasal symptoms. They provide potent anti-inflammatory activity locally at the nasal mucosa while limiting systemic corticosteroid effects ${ }^{3,4}$. They may be used with or without nasal surgery in the treatment of chronic rhinosinusitis with nasal polyps ${ }^{1}$.Mometasonefuroate is a glucocorticosteroid used topically to reduce inflammation of the skin or in the airways. It is a prodrug of the free form mometasone.Fluticasonefuroate is a synthetic, lipophilic, trifluorinatedglucocorticoid receptor agonist. In 2009, Cochrane reviewed the therapeutic effectiveness and adverse event profiles of topical nasal steroids for intermittent and persistent allergic rhinitis in children. In the review, after assessment of trial quality, only three trials involving a total of 79 participants were included in the review (trials were excluded due to the use of 'rescue' medication which the authors state may have confounded the results). All three trials compared topical nasal steroids (Beconase $\AA$ and flunisolide) against placebo. Cochrane concluded that the included trials provided weak and unreliable evidence for the effectiveness of Beconase ${ }^{\circledR}$ and flunisolide for the treatment of allergic rhinitis in children. Until more research is available, decisions on the use of topical steroids should be guided by the physician's clinical experience and patients' individual circumstances and preferences. ${ }^{5}$ FF has the molecular backbone of fluticasone that is also present in fluticasone propionate (FP) but the properties of both molecules are distinctly different ${ }^{6}$. Although FF has a higher receptor affinity and better tissue retention compared to mometasonefuroate $(\mathrm{MF})$, the clinical advantage of FF over MF has not been demonstrated yet in rigorous clinical trials. ${ }^{7,8}$ 
Study Population

\section{Materials And Methods}

Size: 200 Patients

Age Group: 18-75 Years

Place Of Study: Bolung Phc, Lower Dibangvalley, Arunachal Pradesh

Time Period: Between June 2015 - May 2016.

The Study Was A Retrospective Study. The Main Objective Tocompare Clinical effects Between

Mometasonefuroate And Fluticasonefuroate Nasal Spray For Allergic Rhinitis

Although The Study Period Was Short But It Was Important And A Note Should Be Taken About The Actual

Scenario.

\section{Environment}

Communities Having The Same Cultural Practices Are Now Having A Rise In The Incidence Of The Disease. This Is Mainly Due To The Increase In The Populated Households And Ill-Ventilated Houses And Practicing Of Salted Food- Habits.

\section{Clinical Features}

The clinical features can be broadly classified into

Symptoms Signs

Nasal obstruction $100 \%$

Recurrent epistaxis $10 \% \quad$ Nasal cavity mass $\quad 75 \%$

Headache $\quad 40 \% \quad$ Nasal cavity discharge $75 \%$

Smell disorders $\quad 60 \%$

\section{Investigations}

All the cases were diagnosed first by diagnostic nasal endoscopies and then by radiological investigations mainly CT.

\section{Treatment Modalities}

1) Mometasonefuroate : 2 puffs intranasally once daily at bed time for 14 days

2) Fluticasonefuroate: 2 puffs intranasally once daily at bed time for 14 days

\section{Results}

As we can see in table 1.there has been significant changes after treatment but still there is some residual complications which cannot be ignored.

\begin{tabular}{|c|c|c|c|c|c|c|c|c|c|}
\hline COMPLICATIONS & \multicolumn{3}{|c|}{ BEFORE TREATMENT } & \multicolumn{3}{|c|}{ AFTER MOMETASONE TREATMENT } & \multicolumn{3}{|c|}{ APTER PLUTCASONE TREATIESTIT } \\
\hline Nasal cavity obstuction & $\begin{array}{ll}1 & 0\end{array}$ & 0 & $\%$ & 2 & 5 & $\%$ & 3 & 0 & $\%$ \\
\hline $\mathrm{Nas}$ a l d is charge & 7 & 5 & $\%$ & 2 & 5 & $\%$ & 2 & 5 & $\%$ \\
\hline 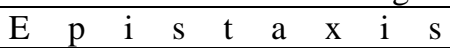 & 1 & 0 & $\%$ & 5 & & $\%$ & 7 & & $\%$ \\
\hline $\mathrm{S}$ me $1 \mathrm{l}$ d i s o r d e r s & 6 & 0 & $\%$ & 2 & 0 & $\%$ & 2 & 8 & $\%$ \\
\hline $\begin{array}{llllllll}\mathrm{H} & \mathrm{e} & \mathrm{a} & \mathrm{d} & \mathrm{a} & \mathrm{c} & \mathrm{h} & \mathrm{e}\end{array}$ & 4 & 0 & $\%$ & 1 & 5 & $\%$ & 1 & 5 & $\%$ \\
\hline
\end{tabular}

Table 1: Decrease In The Rate Of Complications

Post Treatment Complications : A few complications such as unusual/extreme tiredness, weight loss, headache, swellingankles/feet, increased thirst/urination were seen in some of the cases which were treated accordingly.

\section{Discussion}

Nan Zhang et al in their study have shown that a typical clinical situation would be the initiation of drug therapy after allergen exposure or onset of symptoms. ${ }^{9}$ This setting was mimicked by a post-challenge incubation with a concentration range of $10^{-8}$ to $10^{-11} \mathrm{M}$ FF and MF. We observed a concentration-dependent inhibition of cytokine release, which was stronger for FF compared to MF. The maximal inhibition achieved by FF was statistically significantly higher than for MF, which did not suppress IFN- $\gamma$ and TNF- $\alpha$. The experimental setting can be reversed to a pre-challenge exposure resembling a prophylactic use of drugs. In this setup the corticosteroids were pre-incubated with the nasal polyp tissue fragments for one hour before SEB was supplemented. Under these conditions, the maximal cytokine inhibition was not significantly different between FF and MF, but FF consistently inhibited cytokine release at lower concentrations compared to MF. At concentrations as low as $10^{-10} \mathrm{M}$, FF showed a significantly higher suppressive effect versus IL-2, IL-17 and 
IFN- $\gamma$ secretion compared to MF. Interestingly, when the extent of inhibitory effects in this pre- versus the postchallenge exposure setting are compared, it is striking that FF produced maximal inhibition of IL-2, IL-5, IL-17, IFN- $\gamma$ and TNF- $\alpha$ in comparable magnitudes in both experimental setups. In contrast, the maximal inhibition of cytokine release by MF was compromised in the post-challenge incubation setting.In a clinical context, any excess drug that is not taken up into the nasal tissue cells will be rapidly removed by mucociliary clearance within about $30 \mathrm{~min}^{10}$. This was mimicked by washing the tissue in our setup. Subsequently, exposure with SEB was either performed immediately after washing off excess glucocorticoids $(t=0)$, or after another 6 or 24 hours, respectively. Generally, FF inhibited the cytokine release significantly stronger and at earlier time points than MF. Especially the release of IL-5 and TNF- $\alpha$ was most effectively suppressed even by low concentrations of FF and irrespectively of the time interval between removal of the drug excess and exposure with SEB. Thus, the inhibitory activity of FF fully unfolded after only one hour incubation with the drug and was not attenuated over the evaluated time interval.The overall more pronounced anti-inflammatory activity of FF compared to MF is highly consistent with the higher relative receptor affinity (RRA) of FF compared to MF ${ }^{11,12}$.In our experimental setting the nasal mucosal cells were in contact with the glucocorticoids typically for one hour, with the readout performed 24-48 hours later; it can be assumed that classical effects on transcription and translation of genes encoding inflammatory mediators were responsible for the observations ${ }^{13}$. Although an inhibition of inflammatory mediators is the prerequisite for clinical symptom management in allergic rhinitis, the required magnitude and temporal relation of cytokine suppression and the improvement of symptoms is not definite. Efficacy of intranasal corticosteroids can be expected after 7-8 hours of dosing, but some patients appear to benefit more rapidly, e.g. within the first two hours ${ }^{14,15}$. In case of rapid improvement of clinical symptoms nongenomicglucocorticoid effects may play a role as well.

\section{Conclusion}

The study was physician-evaluated for total nasal symptoms. Overall, mometasonefuroate was at least as effective as fluticasonefuroate at equivalent doses. Mometasonefuroate and fluticasonefuroate adequately controlled symptoms of perennial rhinitis and were well tolerated.

\section{Refrences}

[1]. Kalish L, Snidvongs K, Sivasubramaniam R, Cope D, Harvey RJ. Topical steroids for nasal polyps. Cochrane Database of Systematic Reviews 2012, Issue 12. Art. No.: CD006549. DOI: 10.1002/14651858.CD006549.pub2.

[2]. Clinical Knowledge Summaries. Allergic rhinitis. November 2012. Available at: http://cks.nice.org.uk/allergicrhinitis\#azTab

[3]. McCormack PL, Scott LJ. Fluticasonefuroate: intranasal use in allergic rhinitis. Drugs 2007; 67: 1905-15.

[4]. British National Formulary September 2013. Available at http://www.bnf.org/bnf/index.htm

[5]. Al Sayyad JJ, Fedorowicz Z, Alhashimi D, Jamal A. Topical nasal steroids for intermittent and persistent allergic rhinitis in children. Cochrane Database of Systematic Reviews 2007, Issue 1. Art. No.: CD003163

[6]. Biggadike K (2011) Fluticasonefuroate/fluticasone propionate - different drugs with different properties. Clin Respir J 5: $183-4$. doi: 10.1111/j.1752-699x.2011.00244.x

[7]. Valotis A, Högger P (2007) Human receptor kinetics and lung tissue retention of the enhanced-affinity glucocorticoidfluticasonefuroate. Respir Res 8: 54. doi: 10.1186/1465-9921-8-54

[8]. Baumann D, Bachert C, Högger P (2009) Dissolution in nasal fluid, retention and anti-inflammatory activity of fluticasonefuroate in human nasal tissue ex vivo. Clin Exp Allergy 39: 1540-50. doi: 10.1111/j.1365-2222.2009.03306.x

[9]. NanZhang, Koen Van Crombruggen, Gabriele Holtappels ,FengLan ,Michail Katotomichelakis, Luo Zhang ,Petra Högger ,Claus Bachert Suppression of Cytokine Release by FluticasoneFuroate vs. MometasoneFuroate in Human Nasal Tissue ExVivoPublished: April 7, 2014 http://dx.doi.org/10.1371/journal.pone.0093754

[10]. Marttin E, Schipper NG, Verhoef JC, Merkus FW (1998) Nasal mucociliary clearance as a factor in nasal drug delivery. Adv Drug Deliv Rev 29: 13-38. doi: 10.1016/s0169-409x(97)00059-8

[11]. Valotis A, Högger $P$ (2007) Human receptor kinetics and lung tissue retention of the enhanced-affinity glucocorticoidfluticasonefuroate. Respir Res 8: 54. doi: 10.1186/1465-9921-8-54

[12]. Valotis A, Neukam K, Elert O, Högger P (2004) Human receptor kinetics, tissue binding affinity, and stability of mometasonefuroate. J PharmSci 93: 1337-50. doi: 10.1002/jps.20049

[13]. Munck A, Holbrook NJ (1984) Glucocorticoid-receptor complexes in rat thymus cells. Rapid kinetic behavior and a cyclic model. J BiolChem 259: 820-31.

[14]. Hellings PW, Fokkens WJ, Akdis C, Bachert C, Cingi C, et al. (2013) Uncontrolled allergic rhinitis and chronic rhinosinusitis: where do we stand today? Allergy 68(1): 1-7. doi: 10.1111/all.12040

[15]. Katial RK, Salapatek AM, Patel P (2009) Establishing the onset of action of intranasal corticosteroids: is there an ideal study design? Allergy Asthma Proc 30: 595-604. doi: 10.2500/aap.2009.30.3291 